\title{
High Bit Rate Generation of Low Chirped Pulses from Vertical-Cavity Surface-Emitting Lasers via External Axial Magnetic Field
}

\author{
Han van der Lem ${ }^{1}$, J. Martín-Regalado, S. Balle ${ }^{2}$, and Claudio R. Mirasso \\ Departament de Física, Campus UIB, E-07071 Palma de Mallorca, Spain. E-mail: claudio@hp1.uib.es \\ ${ }^{1}$ Permanent Address: Department of Physics and Astronomy, Vrije Universiteit \\ De Boelelaan 1081, $1081 \mathrm{HV}$ Amsterdam, The Netherlands \\ ${ }^{2}$ Also at: Instituto Mediterraneo de Estudios Avanzados, IMEDEA (CSIC-UIB)
}

The possibility of generating low chirped, fast optical pulses with linear polarization in vertical-cavity surfaceemitting lasers is numerically explored. We show that, under the influence of an axial magnetic field, polarization modulation at $\mathrm{GHz}$ rates can be achieved with possible applications in optical clock generation/extraction and optical communications.

Vertical-Cavity Surface-Emitting Lasers (VCSELs) are potentially low-cost sources for future fiber-optic and space communications, optical interconnection, optical computing, optical parallel processing systems, etc., because of their potential capability for ultralow threshold current operation, efficient coupling into optical fibers, large-scale of integration into two-dimensional arrays, and high modulation speed. Transmissions at $10 \mathrm{~Gb} / \mathrm{s}$ for $980 \mathrm{~nm}^{1}$ and $2.5 \mathrm{~Gb} / \mathrm{s}$ for $1.54 \mu \mathrm{m}^{2}$ VCSELs have been recently obtained by direct modulation of the injection current. However, these systems do not take advantage of the polarization properties of these devices which may lead to, e.g. chirp reduction, improvement of the Signal-to-Noise Ratio (SNR), duplication of the transmission capability, etc.

VCSELs are reported to emit linearly polarized light preferentially oriented along either the [110] and [110] crystalline axes of the wafer. The orthogonally polarized modes are frequency non-degenerated as a consequence of the birefringency of the crystal. Although the polarization state of the emitted light can change from one VCSEL to another on the same wafer as the current is varied, polarization stability and polarization switching are the more commonly reported phenomena. Optical pulses with alternating polarization have been obtained, at a maximum modulation rate of $\sim 50 \mathrm{MHz}$, by modulating the injection current around the switching point $^{3}$. This limitation in the maximum frequency could be due to either the thermal response of the device ${ }^{3}$, or to spatial-hole burning ${ }^{4}$. Polarization self-modulation at $\mathrm{GHz}$ rates has also been observed by means of external optical feedback-induced polarization locking ${ }^{5}$.

In this letter we numerically explore an alternative possibility of generating fast optical polarized pulses at $\mathrm{GHz}$ rates by applying an axial magnetic field to the $\mathrm{VC}$ SEL. The magnetic field induces a circular birefringency in the VCSEL so that the output light becomes elliptically polarized ${ }^{6,7}$, as has been experimentally reported in ref. [7]. Here, we show that, when the magnetic-induced circular birefringency is larger than the linear birefringency, emission may occur in an almost rotating linearly polarized (RLP) state $^{6}$ for which the total output power is constant in time, but the power in each orthogonal polarization is modulated at a frequency which depends on the strength of the external magnetic field ${ }^{8}$.

Polarization state selection in VCSELs is commonly explained through the relative red-shift of the gain peak with respect to the linearly polarized resonances due to current-induced thermal heating ${ }^{9}$. However, a recent experiment has shown that polarization switching is present even when the temperature of the active region is kept constant ${ }^{10}$. This behavior is predicted in ref. [11] on the basis of a fundamental model ${ }^{12}$ that considers the coupling of the vector optical field to the allowed transitions between the conduction band and the heavy hole band in unstrained quantum-well VCSELs. The two possible transitions lead to circularly right or left polarized photons, and consequently to two circularly polarized emission channels whose populations, $\mathrm{N}_{ \pm}$, are coupled via spin-flip relaxation processes. In the circularly polarized $\vec{\sigma}_{ \pm}$basis, the rate equation model describing such a system reads ${ }^{6}$ :

$$
\begin{aligned}
& \frac{d E_{ \pm}}{d t}=\kappa(1+i \alpha)(N \pm n-1) E_{ \pm} \\
&-\left(\gamma_{a}+i \gamma_{p}\right) E_{\mp} \pm i \gamma_{z}(B) E_{ \pm}, \\
& \frac{d N}{d t}=-\gamma[(N-\mu)+(N+n)\left|E_{+}\right|^{2} \\
&\left.+(N-n)\left|E_{-}\right|^{2}\right], \\
& \frac{d n}{d t}=-\gamma_{s} n-\gamma\left[(N+n)\left|E_{+}\right|^{2}-(N-n)\left|E_{-}\right|^{2}\right],
\end{aligned}
$$

where $E_{+}$are the slowly varying amplitudes of the circularly polarized components of the vector optical field, $N=N_{+}+N_{-}$is the total population difference between the conduction and the valence bands, and $n=N_{+}-N_{-}$ is the population difference between the two emission channels. In these equations, $\mu$ is the normalized injection current, $\alpha$ is the linewidth enhancement factor, $\kappa$ is the photon decay rate, $\gamma$ is the inverse carrier lifetime, and $\gamma_{s}$ is the spin flip relaxation time. Regarding the anisotropies of the VCSEL, $\gamma_{p}$ and $\gamma_{a}$ represent the linear birefringence and dichroism respectively, and $\gamma_{z}$ represents the magnetic-induced circular birefringency which depends linearly on the strength of the magnetic field, $\mathrm{B}, \mathrm{as}^{7} d \gamma_{z} / d B=8.3 \mathrm{~ns}^{-1} \mathrm{~T}^{-1}$.

For a perfect isotropic VCSEL, $\gamma_{p}=\gamma_{a}=0$, the model predicts stable RLP emission ${ }^{8}$

$$
\left.\vec{E}=\sqrt{\mu-1}\left[e^{i\left(\gamma_{z} t+\psi\right)} \vec{\sigma}_{+}+e^{-i\left(\gamma_{z} t+\psi\right)}\right) \vec{\sigma}_{-}\right],
$$

which, in the linearly polarized $\vec{\sigma}_{x}$ basis, read

$$
\vec{E}=\sqrt{\mu-1}\left[\cos \left(\gamma_{z} t+\psi\right) \vec{\sigma}_{x}+\sin \left(\gamma_{z} t+\psi\right) \vec{\sigma}_{y}\right],
$$

with $\psi$ being an arbitrary angle set to zero for convenience. Thus, the emitted power in each polarization is given by 


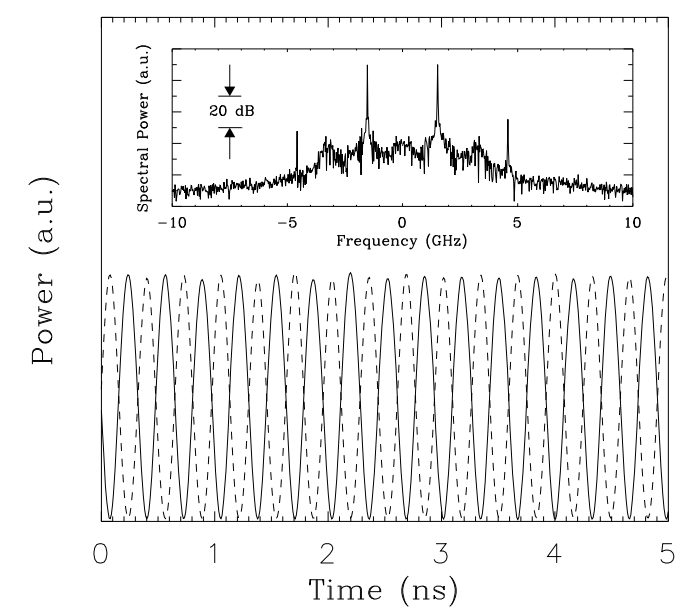

FIG. 1. Output power for the $\hat{x}$ (solid) and $\hat{y}$ (dashed) linearly polarized components corresponding to a rotating elliptically polarized state at $1.2 \mu_{t h}$. The inset shows the optical spectrum of this polarization state. Parameters: $\gamma=$ $1 \mathrm{~ns}^{-1}, \kappa / \gamma=300, \gamma_{s} / \gamma=100, \alpha=4, \gamma_{p} / \gamma=2, \gamma_{a} / \gamma=0.1$, and $\gamma_{z} / \gamma=9.42$.

$$
P_{\substack{x \\ y}}=\left|E_{\substack{x \\ y}}\right|^{2}=\frac{\mu-1}{2}\left[1 \pm \cos \left(2 \gamma_{z} t\right)\right]
$$

Thus, modulation of the output power at $2 \gamma_{z}$ occurs in each linear polarization.

However, for nearly isotropic VCSELs, such that $\gamma_{z} \gg$ $\gamma_{p}, \gamma_{a}$, the RLP state becomes rotating elliptically polarized (REP) but with a very small remanent ellipticity ${ }^{6}$. Nevertheless, modulation of the polarized output still occurs as inferred from Fig. 1, where we plot the output power for both polarizations as a function of time (solid (dashed) line stand for $\hat{x}(\hat{y})$ polarization), obtained from numerical integration of eqs. (1)-(3). Very clear optical pulses are obtained at a rate of $\sim 3 \mathrm{GHz}$ for each linear polarization. This periodically modulated output can be used for optical clock generation without requiring high speed electronics. An additional advantage of this laser system is that each polarization can be independently used to generate a signal by means of, e.g., an external electro-optical modulator, so the amount of information can be duplicated. The inset in Fig. 1 shows the optical spectrum of the REP state, after including Langevin noise terms to eqs. (1)-(3). The two main peaks, at frequencies at -1.5 and $1.5 \mathrm{GHz}$, correspond to the emission frequencies of the circularly polarized left and right states respectively, which form the RLP solution (see Eq. (4)). The additional side bands come as a consequence of the residual linear anisotropies.

Another important feature that comes out from our analysis is that, since the REP solution is a steady state of the system for a fixed value of the injection current (and consequently the carriers are clamped to the threshold), the pulses so obtained have no transient chirp. When comparing with, for example, gain-switched pulses (with a transient chirp of $\sim 20-50 \mathrm{GHz}$ or more), it is clear that our proposed scheme looks specially attractive for optical communication systems.

The rate at which pulses are generated mainly depends on the strength of the magnetic field. Fig. 2

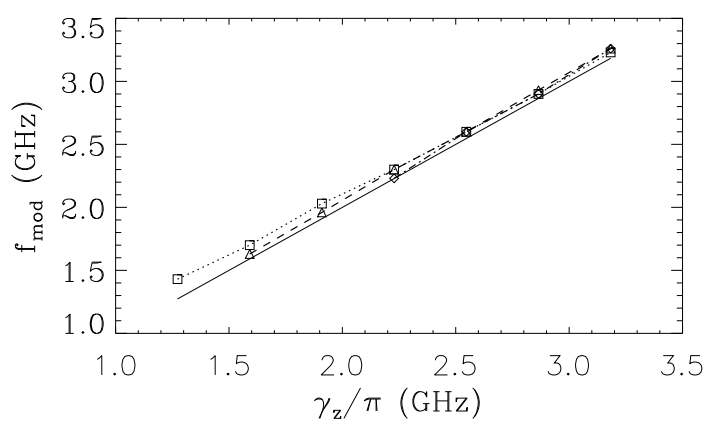

FIG. 2. Dependence of the pulse frequency $\left(f_{p}\right)$ on the magnetically-induced circular birefringency $\left(\gamma_{z}\right)$ for different values of the applied current: 1.2 (squares), 1.5 (triangles), and $1.8 \mu_{t h}$ (diamonds). Same parameters as in Fig. 1.

shows the frequency of the pulsations, $f_{\text {mod }}$, versus the circular birefringence for three different bias currents: 1.2 (squares), 1.5 (triangles), and $1.8 \mu_{t h}$ (diamonds), where $\mu_{t h}$ stands for the threshold current. The dotted line stands for perfect isotropic VCSELs for which $f_{\text {mod }}=2 \gamma_{z} /(2 \pi)$. The frequency at which the VCSEL starts to pulse depends on the injection current in such a way that the higher the bias current, the larger the $\gamma_{z}$ to observe pulses. In addition, the presence of small linear anisotropies slightly modifies the linear behavior. All these features strongly depend on the ratio between linear and circular anisotropies in the system.

The linear dependence of the rotation frequency of the REP state on the magnetic field strength opens the possibility of information transmission by modulation of the magnetic field. This scheme is conceptually similar to frequency modulation, but instead of modulating the carrier frequency of the optical field here the rotating frequency of the polarization orientation is modulated. Although the total emitted power remains constant, the modulation of the magnetic field leads to a modulation of the rotation frequency; hence the repetion rate for the pulses in each polarization is modulated. In Fig. 3 we show the results for such a transmission scheme for a $500 \mathrm{Mb} / \mathrm{s}$ pseudo-random NRZ bit stream, where the magnetic-induced circular birefringence is digitally modulated between $4.7 n s^{-1}$ for " 0 " bits and $9.4 n s^{-1}$ for "1" bits, leading to pulsation frequencies $f_{0} \approx 1.5 \mathrm{GHz}$ for " 0 " bits and $f_{1} \approx 3 \mathrm{GHz}$ for " 1 " bits. A portion of the bit stream is shown in trace (a), and the power emitted in the $\hat{x}$ polarization is shown in trace (b). The different pulse repetion rates for the different bits can be clearly seen, which allows for easy recovery of the information just by filtering the signal received from a moderately fast photodetector which monitors the power on one polarization. Trace (c) shows the output obtained by filtering with a second-order Butterworth electrical band-pass filter centered at $f_{1}$. Alternatively, if the central frequency is $f_{0}$, the resulting signal is the negation of the transmitted signal, as shown in trace $(d)$. The SNR can be improved increasing the ratio $f_{1} / f_{0}$ for constant filter order and filter bandwidth, which must be larger than twice the bit modulation in order to properly detect the signal. Notice also that a similar time trace will be obtained in the $\hat{y}$ polarized beam but shifted half a pe- 


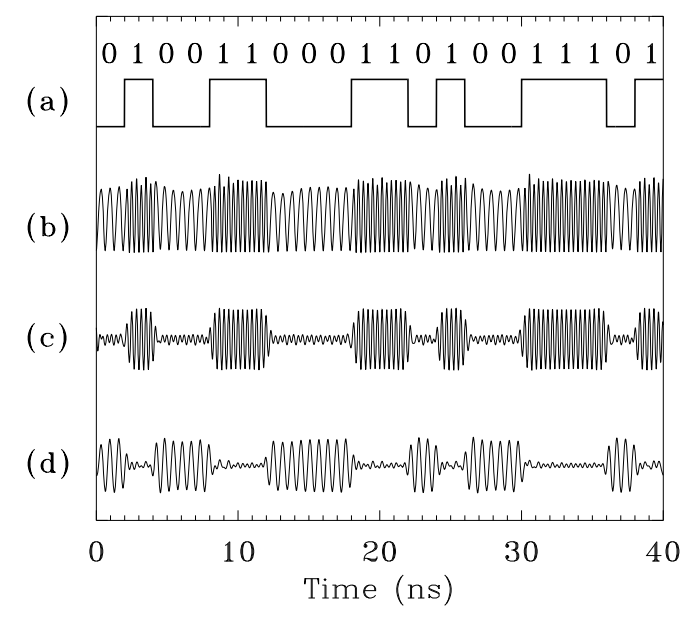

FIG. 3. Digital frequency modulation. (a) bit sequence, (b) optical power emitted in the $\hat{x}$ polarization, (c) signal recovered with a second order Butterworth filter with 1.5 $\mathrm{GHz}$ band-pass centered at $3.1 \mathrm{GHz}$, (d) same as (c) but centered at $1.6 \mathrm{GHz}$. Same parameters as in Fig. 1.

riod. Thus, four copies of the transmitted message can be decoded, which may allow to decrease the bit error rate $(\mathrm{BER})$ in the detector.

In conclusion, we have numerically shown the possibility of generating low-chirp linearly polarized periodic pulses by applying an axial magnetic field to an almost isotropic VCSEL. Gigahertz pulse rates can be achieved with applications in clock generation/extraction, optical communications, optical interconnects, etc. An interesting property of such a laser system is that the pulse frequency mainly depends on the strength of the magnetic field. Based on this dependence, we propose a digital frequency modulation scheme such that information can be easily encoded and decoded.

This work is supported by the projects HCM-CHRXCT94-0594 of the European Union and TIC95/0563 of the Comisión Interministerial de Ciencia y Tecnología CICYT (Spain). Han van der Lem has been supported by the project HCM-CHRX-CT94-0594 of the European Union.

${ }^{1}$ U. Fiedler, G. Reiner, P. Schinetzer and K. J. Ebeling, "Top Surface-Emitting Vertical-Cavity Laser Diodes for $10 \mathrm{~Gb} / \mathrm{s}$ Data Transmission" IEEE Phot. Tech. Lett., vol. 8, p. 746 (1996).

${ }^{2}$ S.Z. Zhang, N.M. Margalit, T.E. Reynolds and J.E. Bowers, "1.54- $\mu \mathrm{m}$ Vertical-Cavity Surface-Emitting Laser Transmission at $2.5 \mathrm{~Gb} / \mathrm{s}$ ", IEEE Phot. Tech. Lett., vol. 9, p. 374 (1997).

${ }^{3}$ K. D. Choquette, K. L. Lear, R. E. Leibenguth and M. T. Asom, "Polarization modulation of cruciform verticalcavity laser diodes", Appl. Phys. Lett. 64, p. 2767 (1994).

${ }^{4}$ A. Valle, L. Pesquera and A. Shore, "Polarization Modulation Dynamics of Birefringent Vertical-Cavity Surface Emitting Lasers", submitted for publication.
${ }^{5}$ S. Jiang, Z. Pan, M. Dagenais, R. A. Morgan and K. Kojima, "High-frequency polarization self-modulation in vertical-cavity surface-emitting lasers", Appl. Phys. Lett., vol. 63, p. 3545 (1993).

${ }^{6}$ C. Serrat, N. B. Abraham, M. San Miguel, R. Vilaseca, and J. Martin-Regalado, "Polarization dynamics in a vertical cavity laser with an axial magnetic field", Phys. Rev. A (Rapid Comm.) 53, R3731 (1996).

${ }^{7}$ A. K. Jansen van Doorn, M. P. van Exter, M. Travagnin, and J. P. Woerdman, "Polarization behavior of surfaceemitting semiconductor lasers in an axial magnetic field", Opt. Commun. 133, p. 252 (1997).

${ }^{8}$ Han van der Lem, "Polarization Effects in a Vertical Cavity Surface Emitting Laser", Master's Thesis, Vrije Universiteit, (Amsterdam, The Netherlands, 1997).

${ }^{9}$ K.D. Choquette, K.L. Lear and R.E. Leibenguth, "Temperature Dependence of Gain-Guided Vertical-Cavity Surface-Emitting Laser Polarization", Appl. Phys. Lett., vol. 64, p. 2062 (1994).

${ }^{10}$ J. Martín-Regalado, J. Chilla, J.J. Rocca and P. Brusenbach, "Polarization Switching in Vertical-Cavity SurfaceEmitting Lasers Observed at Constant Active Region Temperature", Appl. Phys. Lett., vol. 70, (June 1997).

11 J. Martín-Regalado, M. San Miguel, N.B. Abraham, and F. Prati, "Polarization Switching in Quantum Well Vertical-Cavity Surface Emitting Lasers", Optics Letters 21, 765 (1995); "Polarization Properties of Vertical Cavity Surface Emitting Lasers", IEEE J. Quantum Electronics 33, 351 (1996).

${ }^{12}$ M. San Miguel, Q. Feng, J. V. Moloney, "Lightpolarization dynamics in surface-emitting semiconductor lasers", Phys. Rev. A 52, p. 1728 (1995). 